\title{
Genetics of hearing loss: focus on DFNA2
}

\author{
This article was published in the following Dove Press journal: \\ The Application of Clinical Genetics \\ 17 October 2012 \\ Number of times this article has been viewed
}

\section{Laura M Dominguez \\ Kelley M Dodson}

Department of Otolaryngology, Head and Neck Surgery, Virginia Commonwealth University, Richmond,VA
Correspondence: Kelley Dodson Department of Otolaryngology, Head and Neck Surgery, Virginia Commonwealth University, 1200 E Broad St I2, Richmond VA 23298-06|4, USA

$\mathrm{Tel}+\mathrm{I} 8048283965$

Fax + I 8048285779

Email kdodson@mcrh-vcu.edu
Abstract: The purpose of this review is to assess the current literature on deafness nonsyndromic autosomal dominant 2 (DFNA2) hearing loss and the mutations linked to this disorder. Hearing impairment, particularly nonsyndromic hearing loss, affects multiple families across the world. After the identification of the DFNA2 locus on chromosome 1p34, multiple pathogenic mutations in two genes (GJB3 and $K C N Q 4$ ) have been reported. The overwhelming majority of pathogenic mutations linked to this form of nonsyndromic hearing loss have been identified in the $K C N Q 4$ gene encoding a voltage-gated potassium channel. It is believed that KCNQ4 channels are present in outer hair cells and possibly inner hair cells and the central auditory pathway. This form of hearing loss is both phenotypically and genetically heterogeneous and there are still DFNA2 pedigrees that have not been associated with changes in either GJB3 or KCNQ4, suggesting that a possible third gene exists at this locus. Further studies of the DFNA2 locus will lead to a better understanding of progressive hearing loss and provide a better means of early detection and treatment.

Keywords: deafness nonsyndromic autosomal dominant 2, nonsyndromic deafness, genetic hearing loss, GJB3, KCNQ4

\section{Introduction}

Hearing loss is one of the most common sensory deficits seen in the population. Approximately one in 1000 people in the US is born deaf or will develop profound deafness in early childhood. ${ }^{1}$ It is estimated that more than $50 \%$ of cases of childhood deafness are genetic, while the rest are a result of environmental factors. ${ }^{1}$ Certain forms of progressive hearing loss, such as presbycusis, are likely a result of the combination of genetic factors and environmental insults.

Hearing impairment can occur in isolation (nonsyndromic) or in combination with other defects (syndromic). Approximately $70 \%$ of inherited hearing disorders are estimated to be nonsyndromic in nature. ${ }^{1}$ Nonsyndromic hearing loss has a variety of inheritance patterns, including X-linked deafness (DFN), autosomal dominant (DFNA), and autosomal recessive (DFNB) patterns. Generally, recessive traits present with severe hearing loss at an early age, while autosomal dominant traits present as slowly progressive hearing loss later in life.

A systematic literature review of DFNA2 hearing loss was conducted utilizing the US National Library of Medicine Medline database. Over 50 relevant articles relating to DFNA2-associated hearing loss were identified and critically evaluated using evidencebased techniques. All articles with significant contributions to this topic were included in the review, beginning with the first articles in the late 1990s by Xia and Kubisch., ${ }^{34}$ submit your manuscript | www.dovepress.com

Dovepress

http://dx.doi.org/10.2147/TACG.S35525 
To date, over 50 loci and nearly 30 genes have been identified for autosomal dominant nonsyndromic hearing loss, while there are over 100 gene loci associated with all subtypes of nonsyndromic deafness. DFNA2 hearing loss affects numerous families from diverse backgrounds, including those of European, American, and Asian origins, thus demonstrating its genetic heterogeneity. ${ }^{2}$ Two genes have been linked to the DFNA2 locus and some have postulated the existence of a third causative gene. ${ }^{3-5}$ However, pathogenic mutations in the $\mathrm{KCNQ} 4$ gene appear to represent all of DFNA2 associated-hearing impairment, outside of two Chinese families associated with GJB3 mutations. ${ }^{3}$ Genes associated with most forms of autosomal dominant hearing loss have been linked to only a single or few families; however, due to the global impact of DFNA2 mutations, it has been the subject of much interest in recent years. ${ }^{1}$ Kubisch and Xia reported the first mutations in families with DFNA2 hearing loss, and since that time, a wide variety of pathogenic mutations in $K C N Q 4$ have been described in many families. ${ }^{3,4}$ It is anticipated that a better understanding of the mechanism of DFNA2 loss could serve as a potential model for the detection and treatment of progressive hearing loss.

\section{Clinical presentation}

DFNA2-associated hearing loss has a presentation that is typically later in onset and progressive over time, as opposed to early onset and severe loss in recessive forms. High frequency hearing loss may be detected in early childhood, and typically progresses to reach moderate to mid frequency levels of 40-60 decibels (dB) at about 50 years of age, and can exceed $80 \mathrm{~dB}$ at $1 \mathrm{kHz}$ in later years, causing significant problems with speech discrimination in affected patients. ${ }^{6,7}$ Most individuals suffer from severe to profound hearing loss by the age of 70 years, and most are fitted with hearing aids by early adulthood. ${ }^{6}$ Studies of other affected families have shown an annual threshold increase of about $1 \mathrm{~dB}$ per year, with the higher frequencies $(1-8 \mathrm{kHz})$ exhibiting significantly more rapid progression than the lower frequencies $(0.25-0.5 \mathrm{kHz}){ }^{8}$ Other studies have demonstrated similar rates of progression, but no significant evidence of vestibular dysfunction. ${ }^{9}$ One study found a small number of DFNA2 patients (three cases) with vestibular hyperreactivity who were prone to motion sickness; however, the remaining individuals were free of balance issues. ${ }^{7}$ Temporal bone imaging is unremarkable, and physical examination is usually normal. Family history reveals a dominant pattern of inheritance of hearing impairment. The age at initial presentation and progression of hearing loss has shown variability between
DFNA2 families, reinforcing the phenotypic heterogeneity of the disease.

\section{Identification of DFNA2 locus}

Identification of the locus associated with DFNA2 hearing loss was accomplished through the study of a large Indonesian family with over 100 members affected by nonsyndromic progressive autosomal dominant hearing loss. Members with the impairment noted decreased hearing in the second or third decades of life, and audiometry revealed that all affected members had a loss of at least $30 \mathrm{~dB}$ by 30 years of age, with profound loss within 10 years of onset. ${ }^{10}$

Genetic linkage analysis was performed in this Indonesian family as well as in an American and Dutch family with similar audiometric characteristics. First attempts to link the Indonesian family with previously described loci for hereditary deafness on chromosome $5 q$ and $13 q$ were unsuccessful, so microsatellite markers were used to search the entire genome. Linkage was ultimately detected on the short arm of chromosome 1, also known as 1p34. Association with this locus was also confirmed in the American family, but the Dutch family could not be linked to this site, reaffirming that autosomal dominant deafness is not only clinically but also genetically heterogeneous. ${ }^{10}$

\section{Connexins and hearing loss}

Mutations in GJB2 encoding connexin 26, the most common cause of nonsyndromic hearing loss, have previously been shown to cause autosomal recessive (DFNB1) and dominant (DFNA3) forms of hearing impairment. ${ }^{11}$ This mutation, as well as mutant connexin 30, are both found at the DFNA3 locus. ${ }^{12,13}$ Xia et al proposed that other members of the connexin family could be involved in DFNA2 hearing loss, so the gene (GJB3) encoding gap junction protein beta-3 (connexin 31 ) was cloned. ${ }^{3}$ This gene was mapped to chromosome $1 \mathrm{p} 34$.

GJB3 is comprised of two exons, only the second of which is a coding exon. In order to determine if there was any association of connexin 31 with DFNA2 hearing loss, the coding regions of 42 families with hereditary diseases linked to the short arm of chromosome 1 were sequenced. These diseases included sensorineural hearing loss, erythrokeratodermia, Charcot-Marie-Tooth disease, and ptosis. In two small Chinese families with sensorineural hearing impairment, a missense and a nonsense mutation were identified in the coding region of GJB3, while the individuals with the other mentioned disease states did not exhibit any mutations (Table 1). ${ }^{3}$ This was the first identification 
Table I GJB3 mutations

\begin{tabular}{lllll}
\hline Family & Mutation & $\begin{array}{l}\text { Amino acid } \\
\text { change }\end{array}$ & $\begin{array}{l}\text { Amino acid } \\
\text { position }\end{array}$ & Reference \\
\hline Chinese & Missense & $547 \mathrm{G} \rightarrow \mathrm{A}$ & 183 & Xia et a $\left.\right|^{3}$ \\
Chinese & Nonsense & $538 \mathrm{C} \rightarrow \mathrm{T}$ & 180 & ${\text { Xia et a }\left.\right|^{3}}$ \\
\hline
\end{tabular}

of a connexin gene linked to the DFNA2 locus. Nucleotide changes of $G J B 2$ were identified in these families, but these were felt to be polymorphisms unrelated to the hearing loss in this group. ${ }^{3}$

Some controversy exists over the role of GJB3 mutations in DFNA2 hearing loss. Some have stated that the evidence presented supporting the role of $G J B 3$ in nonsyndromic hearing loss is not convincing. ${ }^{14}$ In the Chinese families evaluated, one female carrier of the mutation exhibited normal hearing by audiogram, suggesting incomplete penetrance, which typically is not seen with autosomal dominant forms of hearing loss. ${ }^{3,14}$ Further studies of a five-generation family with a progressive, high frequency autosomal dominant hearing loss revealed a one-sequence alteration in the first noncoding exon of GJB3. However, this mutation was identified in both affected and unaffected family members, again suggesting that GJB3 may not be directly involved in this type of progressive hearing impairment. ${ }^{5}$

Additionally, in 1998, when the two Chinese families with GJB3 mutations were being studied, mutations in the voltagegated potassium channel (KCNQ4), now linked with DFNA2 hearing loss, was yet to be identified. Therefore, it is possible that these families had an existing mutation in the KCNQ4 voltage-gated potassium channel that remained undetected. ${ }^{14}$ To date, no other DFNA2 families have demonstrated the GJB3 mutation. Attention has now shifted towards the elucidation of mutations in voltage-gated ion channels. This has proven to be an extremely prosperous endeavor because various mutations in potassium channels have been linked to hearing loss in families from around the world (Table 2).

\section{Role of potassium channels in hearing loss}

Ion channels have a central role in regulation of the ionic composition of intracellular and extracellular fluid, which are key for hair cell activation in the inner ear. Mutations in these channels have long been suspected to play a part in hearing loss, particularly due to the importance of ion regulation in perilymph and endolymph in the inner ear. Signal transduction in the cochlea depends on maintenance of a high potassium concentration and a positive potential in the endolymph. This is mediated through ion transport across the stria vascularis into the hair cells and efflux of $\mathrm{K}+$, likely through voltage-gated ion channels. ${ }^{15}$ Inner hair cells provide the brain with electrical input while outer hair cells amplify acoustic vibrations. ${ }^{15}$

Mutations leading to human disease including cardiac arrhythmias, epilepsy, and congenital deafness have been found in approximately ten potassium channel genes. Four of these known genes encode $\mathrm{K}+$ channels from the

Table 2 Known KCNQ4 mutations

\begin{tabular}{|c|c|c|c|c|}
\hline Family & Mutation & Protein domain & Exon & Reference \\
\hline Dutch I & W276S & Pore region & 5 & Coucke et $\mathrm{al}^{20}$ \\
\hline Dutch II & G32IS & S6 transmembrane domain & 7 & Coucke et $\mathrm{al}^{20}$ \\
\hline Dutch III & L274H & Pore region & 5 & Van Hauwe et al ${ }^{18}$ \\
\hline Dutch IV & W276S & Pore region & 5 & Van Camp et $\mathrm{al}^{24}$ \\
\hline Dutch V & W276S & Pore region & 5 & Topsakal et a ${ }^{23}$ \\
\hline Belgian* & Fs7IX134 & $\mathrm{N}$-terminal cytoplasmic region & 5 & Coucke et $\mathrm{a}^{20}$ \\
\hline Japanese I & W276S & Pore region & 5 & Akita et $\mathrm{a}^{25}$ \\
\hline Japanese II & W276S & Pore region & 5 & Van Camp et a $\left.\right|^{24}$ \\
\hline Japanese III* & Fs7IX138 & $\mathrm{N}$-terminal cytoplasmic region & 1 & Kamada et a ${ }^{30}$ \\
\hline American I & G285C & Pore region & 6 & Coucke et $\mathrm{al}^{20}$ \\
\hline American II & L28IS & Pore region & 6 & Talebizadeh et $\mathrm{al}^{27}$ \\
\hline French & G285S & Pore region & 6 & Kubisch et $\mathrm{al}^{4}$ \\
\hline Taiwanese & FI82L & S3 Transmembrane domain & 4 & Su et $\mathrm{al}^{29}$ \\
\hline Spanish & G296S & Pore region & 6 & Mencia et $\mathrm{a}^{21}$ \\
\hline American III & G287A & Pore region & 6 & Arnett et $\mathrm{al}^{22}$ \\
\hline Korean & c664_dell8 & S4-S5 intramembrane loop & 4 & Baek et $\mathrm{al}^{17}$ \\
\hline American IV & $\mathrm{E} 260 \overline{\mathrm{K}}$ & S5 transmembrane domain & 5 & Hildebrand et $\mathrm{a}^{31}$ \\
\hline American $\mathrm{V}$ & $\mathrm{D} 262 \mathrm{~V}$ & S5 transmembrane domain & 5 & Hildebrand et $\mathrm{a}^{31}$ \\
\hline American $\mathrm{VI}^{*}$ & W24IX & S5 transmembrane domain & 5 & Hildebrand et $\mathrm{a}^{31}$ \\
\hline
\end{tabular}

Note: *Nonsense mutations resulting in truncated peptide. 
KCNQ family. ${ }^{15}$ Since previous relationships between KCNQ1 mutation and deafness in the Jervell and LangeNielsen syndrome were elucidated, $K C N Q 4$ has been thought to be a candidate gene for individuals with slowly progressive hearing loss. ${ }^{16}$

\section{KCNQ4 structure and function}

In recent years, increased attention has been directed towards identification of mutations and pathogenic mechanisms of the KCNQ4 gene and its role in hearing loss. This is a complex gene with 14 exons encoding a protein of 695 amino acids. It has a long C-terminus and six transmembrane domains with the fourth domain (S4) serving as the voltage sensor segment. The fifth and sixth domains constitute the pore region with the peptides in between the domains composing the P-loop. This single pore region, which forms the selectivity filter of the pore, comprises only $3 \%$ of the KCNQ4 protein, yet is where the majority of mutations linked to nonprogressive autosomal dominant hearing loss have been identified..$^{15,17,18}$

Studies of murine cochlear RNA demonstrate KCNQ4 transcripts in the brain and in the outer hair cells of the cochlea. ${ }^{4,19}$ Inner and outer hair cell apical membranes are in contact with endolymph that fills the scala media which has a high potassium concentration and low sodium concentration. Apical potassium channels lead to an influx of $\mathrm{K}+$ while basolateral channels likely mediate efflux of $\mathrm{K}+$. The location of the KCNQ4 potassium channel on the basal membrane of the outer hair cell prohibits function as a mechanosensitive channel or motor protein. ${ }^{19}$ It is suspected that KCNQ4 contributes to basolateral $\mathrm{K}+$ conductance of the ion and removal of intracellular $\mathrm{K}+$ taken up from the endolymph. ${ }^{4}$ Further studies have suggested that mutation of $\mathrm{KCNQ}_{4}$ may cause chronic $\mathrm{K}+$ overload, causing slow degeneration of outer hair cells, therefore explaining the progressive nature of DFNA2 hearing loss. ${ }^{15}$

\section{Identification of KCNQ4 mutations}

Since the first identification of a $\mathrm{KCNQ} 4$ mutation linked to DFNA2 hearing impairment in 1999, there have been 18 additional families having similar hearing loss with mutations of $\mathrm{KCNQ}_{4}$ (Table 2 and Figure 1). Although this gene has 14 exons, the mutations seem to cluster around exons 4, 5 , 6 , and 7 , with missense mutations being the most common.

Initial studies of the $\mathrm{KCNQ}_{4}$ gene identified a missense mutation $(\mathrm{G} 285 \mathrm{~S})$ in exon 6 in a heterozygous state in three members of a French family with profound hearing loss. ${ }^{4}$ Potassium channel pores have a highly conserved glycine in the GYG K+ channel signature sequence that is found in different classes of channels in all species. This missense mutation, along with another mutation, G285C, identified in an American family, were found to affect the first glycine in this sequence which disrupts the channel selectivity filter and channel function. ${ }^{4,20}$ These mutations prevent the ability of the channel to distinguish between $\mathrm{K}+$ and $\mathrm{Na}+$ ions. ${ }^{16}$ Mutant channels did not have any detectable currents when expressed in Xenopus oocytes,

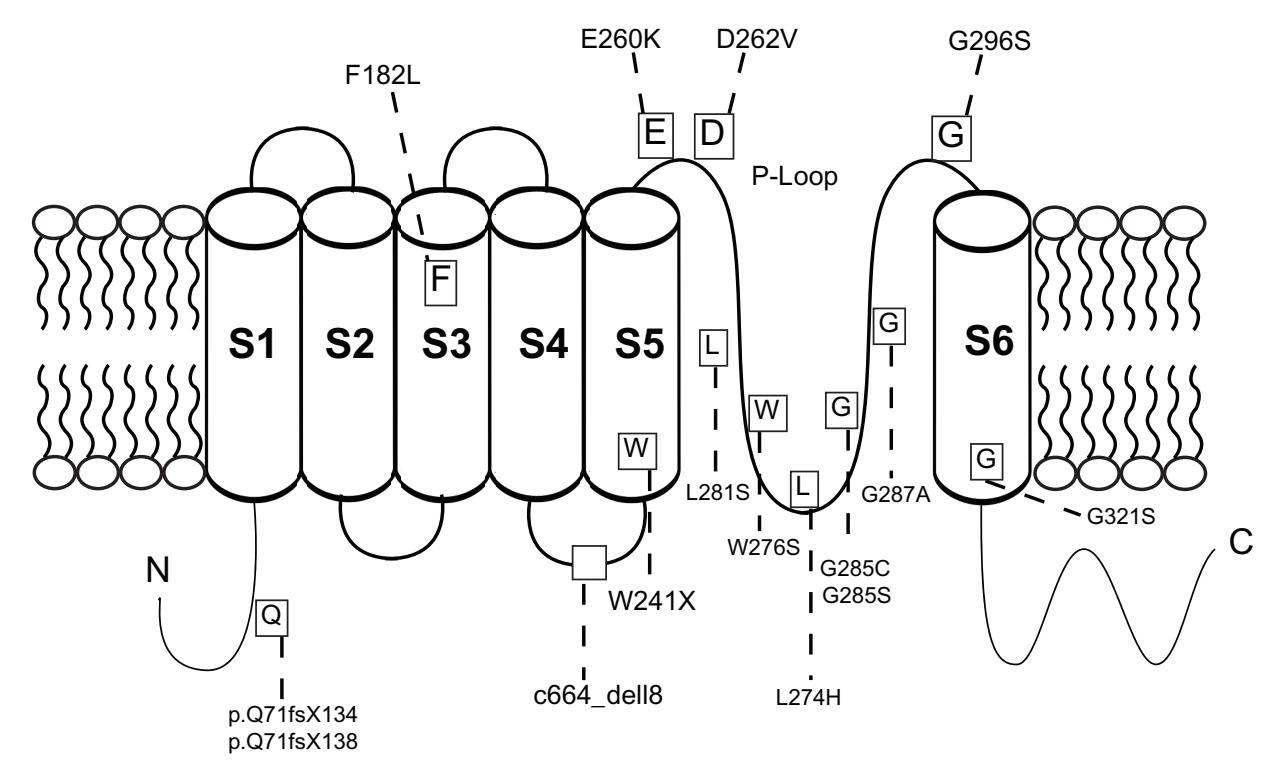

Figure I Structure of KCNQ4.

Notes: Schematic representation of KCNQ4 demonstrating its six transmembrane domains, pore region, and long C-terminus. The labeled boxes represent all previously reported mutations in DFNA2 pedigrees. The majority of mutations cluster around the P-loop. 
and when injected into a wild-type KCNQ4 model to mimic a heterozygous state, there was a significant reduction of channel currents by about $90 \%$. This led to the proposal that mutant $\mathrm{KCNQ} 4$ exerts a dominant negative effect on the wild-type gene. ${ }^{4,15}$

Further analysis of DFNA2 families revealed that the majority of the missense mutations clustered around the pore region. The G296S mutation, located in the pore region, identified in a small Spanish family, also demonstrated a strong dominant negative effect on wild-type channels. ${ }^{21}$ Another missense mutation, G287A, identified by Arnett et al in an American family was also found to disrupt the GYG sequence just as in the initial reported $K C N Q 4$ mutation. ${ }^{22}$

The only missense mutation that has been identified in multiple families of diverse backgrounds is W276S, which has led to the proposal that this represents a mutational hot spot. ${ }^{20,23-25}$ With this mutation, Trp276 is altered. This amino acid is located in the pore loop of KCNQ4 channels adjacent to another highly conserved tryptophan residue which has been proposed to function in maintaining the appropriate diameter of the pore. ${ }^{26}$

Further mutations at the pore region include L281S and L274S identified in an American and Dutch family, respectively. ${ }^{18,27}$ It is proposed that replacing these hydrophobic leucine residues with hydrophilic amino acids disrupts the structure of the KCNQ4 channel. ${ }^{28}$ Another unique mutation in a Dutch family was a G321S mutation at the S6 transmembrane domain, which also contributes to pore structure. ${ }^{20}$ To date, only one mutation in a Taiwanese family has been linked to the S3 transmembrane domain. This represents a missense mutation that is not linked to pore formation. ${ }^{29}$

Thus far, only three mutations resulting in truncated peptides have been elucidated. ${ }^{20,30,31}$ The first FS71 mutation was identified in a Belgian family. Interestingly, this mutation was not in the pore region, but in the N-terminal cytoplasmic region. ${ }^{20}$ The second, found in a Japanese family, was a one-base deletion in exon 1, causing a frameshift mutation with a resultant truncated peptide before the first transmembrane domain. ${ }^{30}$ The third stop codon mutation, identified in an American family on exon 5, is predicted to result in a truncated peptide that lacks most of the S5 transmembrane domain, the S6 domain, and the C-terminal region. ${ }^{31}$

It has been suggested that the type, rather than the location, of the mutation has the greatest effect on patient phenotype. This was demonstrated in a Dutch family with a S6 transmembrane domain missense mutation that exhibited a phenotype similar to that of patients with pore region missense mutations. ${ }^{18,20}$ Individuals with the mutation identified by Kamada et al, ie, a nonsense mutation in the $\mathrm{N}$-terminal cytoplasmic region, had late-onset and pure high frequency hearing loss. ${ }^{30}$ Coucke et al found a similar phenotype in patients with a nonsense mutation in FS71 in the S6 domain, suggesting that deletions in general are associated with later-onset hearing loss that is mild when compared with missense mutations. ${ }^{20}$ However, the stop codon identified by Hildebrand et al did not fit this phenotype, because the affected individual had a more severe form of hearing loss requiring cochlear implantation during childhood. ${ }^{31}$

Analysis of a Korean family identified an 18 nucleotide deletion (c.664_del18) in exon 4 of the $K C N Q 4$ gene which is suspected to result in a six amino acid deletion in the intramembrane loop between the S4 and S5 transmembrane domains. ${ }^{17}$ Human embryonic kidney cells were cotransfected with the wild-type $K C N Q 4$ and the exon 4 deletion, W276S mutation, and G285C mutations. Cells cotransfected with previously identified pore-loop missense mutations, W276S and G285C, as well as the exon 4 deletion exhibited normal protein synthesis and membrane expression of the ion channel, but there was no appreciable ion flow through the membrane. This suggests an inhibition of KCNQ4 channel function through a dominant negative effect and illustrates the importance of the S4-S5 intramembrane loop. ${ }^{17}$

In deletion mutations, haploinsufficiency has been proposed as the pathogenic mechanism of action. ${ }^{30}$ Haploinsufficiency may result from a null mutation, but it is possible that there is still $K C N Q 4$ function from the intact allele. This may provide sufficient KCNQ4 channels to cause only mild hearing impairment, therefore suggesting a genotype-phenotype correlation. ${ }^{30}$ Studies in mice with total $K C N Q 4$ knockout showed a more rapidly progressive hearing loss in comparison with those heterozygous for the dominant negative mutation. Because $\mathrm{K}+$ channels form tetramers, it can be assumed that $1 / 16$ of potassium channels in a functional state can lead to a significant delay in hair cell degeneration. Those heterozygous for the knockout mutation did not show any hearing abnormalities, suggesting that only $50 \%$ of currents are required for normal hearing. ${ }^{32}$

Hearing loss of 50-60 dB in these mice would correlate with a selective loss of outer hair cell function. ${ }^{32}$ However, DFNA2 pedigrees with hearing loss $>60 \mathrm{~dB}$ has led others to 
suggest that outer hair cell dysfunction alone cannot account for the degree of hearing loss seen in these patients. ${ }^{33}$

\section{KCNQ4 not just in outer hair cells}

Different mechanisms of KCNQ4-mediated hearing loss have been proposed, including the previously mentioned $\mathrm{K}+$ recirculation in outer hair cells. ${ }^{4,15}$ Interestingly, it has also been noted that there is higher expression of KCNQ4 potassium channels in the basal turn of the cochlea, which is responsible for high frequency hearing and would thus explain the high frequency hearing loss seen early in patients with DFNA2. ${ }^{4}$

Others have proposed defective signal transmission in the central auditory pathway as a potential cause of progressive hearing loss. ${ }^{19,33}$ In addition to the basolateral membranes of outer hair cells, immunocytochemistry has revealed the presence of KCNQ4 in type 1 vestibular hair cells, as well as several nuclei of the central auditory pathway in the brainstem. ${ }^{19}$ The role of KCNQ4 in vestibular hair cells is unclear because DFNA2 patients do not typically exhibit vestibular symptoms. This was confirmed with further experiments where mice had a constitutive knockout of $K C N Q 4{ }^{32}$

An additional proposed mechanism of KCNQ4 hearing loss centers on dysfunctional basal inner hair cells and spiral ganglion neurons. ${ }^{33}$ The belief that defective outer hair cells cannot account for the progressive nature of hearing impairment in DFNA2 pedigrees led to the study of the distribution of KCNQ4 protein and related it to expression of Kcnq4 splice variants. ${ }^{34}$ Four splice variants were identified, with expression of the third variant (Kcnq4_v3) restricted primarily to the cochlea and with the highest expression in the basal spiral ganglion and inner hair cells. ${ }^{34}$ It is proposed that this pattern of expression could explain the high frequency hearing loss seen in DFNA2 families. ${ }^{34}$ Furthermore, the expression patterns in the murine organ of Corti and spiral ganglion cells have led to the hypothesis that progressive hearing loss could be a result of increasing mutation load toward the apex of the inner hair cells and neurons of the spiral ganglion, resulting in accumulation of defective proteins and, ultimately, a negative effect on cochlear function. ${ }^{34}$

\section{Possible third gene responsible for DFNA2 hearing loss}

To date, two genes have been linked to the DFNA2 locus, ie, GJB3, encoding connexin 31, and $K C N Q 4$, encoding a voltage-gated potassium channel. ${ }^{3,4}$ Controversy does exist over the association between changes found in GJB3 and DFNA2-associated deafness, as described above. Others have proposed that a third gene may exist at this locus which has yet to be identified. An Indonesian family that was mapped to the DFNA2 locus did not have an identifiable mutation in connexin 31 or the KCNQ4 voltage-gated potassium channel, suggesting that there is potentially another causative gene. ${ }^{10,18}$

The UCSF-99 family, a large five-generation family with a pattern of hearing loss characteristic of DFNA2 was linked to chromosome 1p34, the site of the DFNA2 locus. While sequence changes were seen in this family for both $G J B 3$ and $K C N Q 4$, these mutations were seen in both affected and unaffected family members, suggesting that neither gene was truly responsible for the hearing impairment. ${ }^{5}$ This particular family does differ from other DFNA2 pedigrees in that the onset of hearing loss is later and progresses at a slower rate, providing further evidence that the DFNA2 locus is genetically heterogeneous.

Proposed candidates for this unidentified gene include POU $3 F 1$ and COL9A2 which are located within the mapped interval described by Goldstein et al. ${ }^{5}$ Two members of the $P O U$ transcription factor family, which are expressed in embryonal stem cells and the brain, have been implicated in hearing loss. POU3F4 has been associated with DFN3 (deafness with fixation of the stapes) which is the most common X-linked form of hearing impairment, while POU $4 F 3$ has been linked with DFNA15 hearing loss, another form of nonsyndromic hearing loss. ${ }^{35,36}$ This makes POU $3 F 1$ an attractive candidate gene for further analysis.

Collagen proteins have also been linked with hearing impairment, such as the association of COL11A2 with DFNA13 hearing loss which primarily affects the mid frequencies. A connection has also been identified between type IV collagen mutations and Alport syndrome. ${ }^{5}$ COL9A2 will also likely see further attention in the coming years as a possible causal gene in nonsyndromic progressive hearing loss.

Currently, detection of DFNA2 hearing loss is based on characteristic clinical findings, including audiometric characteristics as well as a family history of dominant later-onset progressive hearing loss.

The only definitive means of diagnosing DFNA2associated hearing impairment is molecular genetic testing. Through elucidation of the pathogenic mechanisms of DFNA2-associated hearing impairment, early diagnosis and eventually gene therapy for this condition will hopefully become available. In addition, this could potentially 
serve as a model for further study of more common forms of progressive hearing loss.

\section{Conclusion}

DFNA2-associated hearing loss is both clinically and genetically heterogeneous. To date, two genes, $K C N Q 4$ and GJB3, have been linked to the DFNA2 locus on the short arm of chromosome 1 with most, if not all, pathogenic mutations identified in KCNQ4. Due to the inability to link certain DFNA2 families to mutations of GJB3 or KNCQ4, it has been proposed that a third causative gene exists. The majority of focus has been directed towards mutations of the KCNQ4 voltage-gated ion channel, and a variety of missense and nonsense mutations have been identified in families from diverse backgrounds. With the large population that is affected by hearing loss, it is presumed that a better understanding of the molecular mechanisms of DFNA2 hearing impairment will lead to improved methods of diagnosis and, potentially, treatment of this condition, as well as other forms of progressive hearing loss.

\section{Acknowledgment}

We would like to thank Kevin A Heraldo for assistance with graphics.

\section{Disclosure}

The authors report no conflicts of interest in this work.

\section{References}

1. Morton NE. Genetic epidemiology of hearing impairment. Ann NYAcad Sci. 1991;630:16-31.

2. Van Camp G, Smith RJH. Hereditary hearing loss. Available from: http:// hereditaryhearingloss.org. Accessed April 11, 2012.

3. Xia JH, Liu CY, Tang BS, et al. Mutations in the gene encoding gap junction protein beta-3 associated with autosomal dominant hearing impairment. Nat Genet. 1998;20:370-373.

4. Kubisch C, Schroeder BC, Friedrich T, et al. KCNQ4, a novel potassium channel expressed in sensory outer hair cells, is mutations in dominant deafness. Cell. 1999;96:437-446.

5. Goldstein JA, Lalwani AK. Further evidence for a third deafness gene within the DFNA2 locus. Am J Med Genet. 2002;108:304-309.

6. DeLeenheer EM, Ensink RJ, Kunst HP, et al. DFNA2/KCNQ4 and its manifestations. Adv Otorhinolaryngol. 2002;61:41-46.

7. De Leenheer EM, Huygen PL, Couke PJ, Admiraal RJ, Van Camp G, Cremers CW. Longitudinal and cross-sectional phenotype analysis in a new, large Dutch DFNA2/KCOQ4 family. Ann Otol Rhinol Laryngol. 2002;111:267-274.

8. Kunst H, Marres H, Huygen P, et al. Nonsyndromic autosomal dominant progressive sensorineural hearing loss: audiologic analysis of a pedigree linked to DFNA2. Laryngoscope. 1998;108:74-80.

9. Marres H, van Ewijk M, Huygen P, et al. Inherited nonsyndromic hearing loss. An audiovestibular study in a large family with autosomal dominant progressive hearing loss related to DFNA2. Arch Otolaryngol Head Neck Surg. 1997;123:573-577.
10. Coucke PJ, Van Camp G, Djoyodiharjo B, et al. Linkage of autosomal dominant hearing loss to the short arm of chromosome 1 in two families. N Engl J Med. 1994;331:425-431.

11. Denoyelle F, Lina-Granade G, Plauchu H, et al. Connexin 26 gene linked to a dominant deafness. Nature. 1998;393:319-320.

12. Kelsell DP, Dunlop J, Stevens HP, et al. Connexin 26 mutations in hereditary nonsyndromic sensorineural deafness. Nature. 1997;387: 80-83.

13. Grifa A, Wagner CA, D’Ambrosio L, et al. Mutations in GJB6 cause nonsyndromic autosomal dominant deafness at DFNA3 locus. Nat Genet. 1999;23:16-18.

14. Smith RJ, Hildebrand M. DFNA2 nonsyndromic hearing loss. In: Gene Reviews at Gene Tests: Medical Genetics Information Resources (database online). Available from: http://www.genetests.org. Updated February 17, 2011. Accessed April 11, 2012.

15. Jentsch TJ. Neuronal KCNQ potassium channels: physiology and role in disease. Nat Rev Neurosci. 2000;1:21-30.

16. Neyroud N, Tesson F, Denjoy I, et al. A novel mutation in the potassium channel gene KVLQT1 causes the Jervell and Lange-Nielsen cardioauditory syndrome. Nat Genet. 1997;15:186-189.

17. Baek JI, Park HJ, Park K, et al. Pathogenic effects of a novel mutation (c.664_681del) in KCNQ4 channels associated with auditory pathology. Biochim Biophys Acta. 2011;1812:536-543.

18. Van Hauwe P, Couke PJ, Ensink RJ, Huygen P, Cremers CW, Van Camp G. Mutations in the KCNQ4 K+ channel gene, responsible for autosomal dominant hearing loss, cluster in the channel pore region. Am J Med Genet. 2000;93:184-187.

19. Kharkovets T, Hardelin JP, Safieddine S, et al. KCNQ, a K+ channel mutated in a form of dominant deafness, is expressed in the inner ear and the central auditory pathway. Proc Natl Acad Sci USA. 2000;97(8):4333-4338.

20. Coucke PJ, Van Hauwe P, Kelley PM, et al. Mutations in the KCNQ4 gene are responsible for autosomal dominant deafness in four DFNA2 families. Hum Mol Genet. 1999;8:1321-1328.

21. Mencia A, Gonzalez-Nieto D, Modamio-Hoybjor S, et al. A novel mutation KCNQ4 pore-region mutation (p.G296S) causes deafness by impairing cell-surface channel expression. Hum Genet. 2008;123:41-53.

22. Arnett J, Emery SB, Kim TB, et al. Autosomal dominant progressive sensorineural hEaring loss due to a novel mutation in the KCNQ4 gene. Arch Otolaryngol Head Neck Surg. 2011;137:54-59.

23. Topsakal V, Pennings RJ, te Brinke H, et al. Phenotype determination guides swift genotyping of a DFNA2/KCNQ4 family with a hot spot mutation (W276S). Otol Neurotol. 2005;26:52-58.

24. Van Camp G, Couke PJ, Akita J, et al. A mutational hot spot in the KCNQ4 gene responsible for autosomal dominant hearing impairment. Hum Mutat. 2002;20:15-19.

25. Akita, J, Abe S, Shinkawa H, Kimerling WJ, Usami S. Clinical and genetic features of nonsyndromic autosomal dominant sensorineural hearing loss: KCNQ4 is a gene responsible in Japanese. J Hum Genet. 2001;46:355-361.

26. Doyle DA, Morais Cabral J, Pfuetzner RA, et al. The structure of the potassium channel: molecular basis of $\mathrm{K}+$ conduction and selectivity. Science. 1998;280:69-77.

27. Talebizadeh Z, Kelley PM, Askew JW, Beisel KW, Smith SD. Novel mutation in the KCNQ4 gene in a large kindred with dominant progressive hearing loss. Hum Mutat. 1999;14:493-501.

28. Nie L. Mutations in KCNQ4 channels associated with nonsyndromic progressive sensorineural hearing loss. Curr Opin Otlolaryngol Head Neck Surg. 2008;16:441-444.

29. Su CC, Yang JJ, Shieh JC, Su MC, Li SY. Identification of novel mutations in the KCNQ4 gene of patients with nonsyndromic deafness from Taiwan. Audiol Neurolotol. 2007;12:20-26.

30. Kamada F, Kure S, Kudo T, et al. A novel KCNQ4 one-base deletion in a large pedigree with hearing loss: implication for the genotypephenotype correlation. J Hum Genet. 2006;51:455-460. 
31. Hildebrand MS, Tack D, McMordie SJ, et al. Audioprofile-directed screening identified novel mutations in KCNQ4 causing hearing loss at the DFNA2 locus. Genet Med. 2008;10:797-804.

32. Kharkovets T, Dedek K, Maier H, et al. Mice with altered KCNQ4 K+ channels implicate sensory outer hair cells in human progressive deafness. EMBO J. 2006;25:642-652.

33. Beisel KW, Nelson NC, Delimont DC, Fritzsch B. Longitudinal gradients of KCNQ4 expression in spiral ganglion and cochlear hair cells correlate with progressive hearing loss in DFNA2. Brain Res Mol Brain Res. 2000;82:137-149.
34. Beisel KW, Rocha-Sanchez SM, Morris KA, et al. Differential expression of KCNQ4 in inner hair cells and sensory neurons is the basis of progressive high-frequency hearing loss. J Neurosci. 2005;25: 9285-9293.

35. De Kok YJJ, van der Maaerl SM, Bitner-Glindzicz M, et al. Association between X-linked mixed deafness and mutations in the POU domain gene POU3F4. Science. 1995;267:685-688.

36. Vahava O, Morell R, Lynch ED, et al. Mutations in transcription factor POUF43 associated with inherited progressive hearing loss in humans. Science. 1998;279:1950-1954.

\section{Publish your work in this journal}

The Application of Clinical Genetics is an international, peer-reviewed open access journal that welcomes laboratory and clinical findings in the field of human genetics. Specific topics include: Population genetics; Functional genetics; Natural history of genetic disease; Management of genetic disease; Mechanisms of genetic disease; Counselling and ethical issues; Animal models; Pharmacogenetics; Prenatal diagnosis; Dysmorphology. The manuscript management system is completely online and includes a very quick and fair peer-review system, which is all easy to use. Visit http://www.dovepress.com/testimonials.php to read real quotes from published authors.

Submit your manuscript here: http://www.dovepress.com/the-application-of-clinical-genetics-journal 